\section{Reflux and cough}

\section{S29 COUGH FREQUENCY AND MORBIDITY IN INPATIENTS WITH ACUTE RESPIRATORY DISEASE}

${ }^{1}$ RD Turner, ${ }^{2}$ SS Birring, ${ }^{3}$ S Matos, ${ }^{1} \mathrm{GH}$ Bothamley; ${ }^{1}$ Homerton University Hospital NHS Foundation Trust, London, UK; ${ }^{2}$ King's College Hospital NHS Foundation Trust, London, UK; ${ }^{3}$ Institute of Electronics and Telematics Engineering, University of Aveiro, Aveiro, Portugal

\subsection{6/thoraxjnl-2013-204457.36}

Introduction Cough is the unique respiratory symptom. Although associated with a range of conditions it has been little studied in acute respiratory disease. We describe cough frequency and cough-related quality of life in this group.

Method Participants had a diagnosis of acute exacerbation of asthma (asthma), chronic obstructive pulmonary disease (AECOPD), or lower respiratory tract infection (including community-acquired pneumonia) in the absence of the other
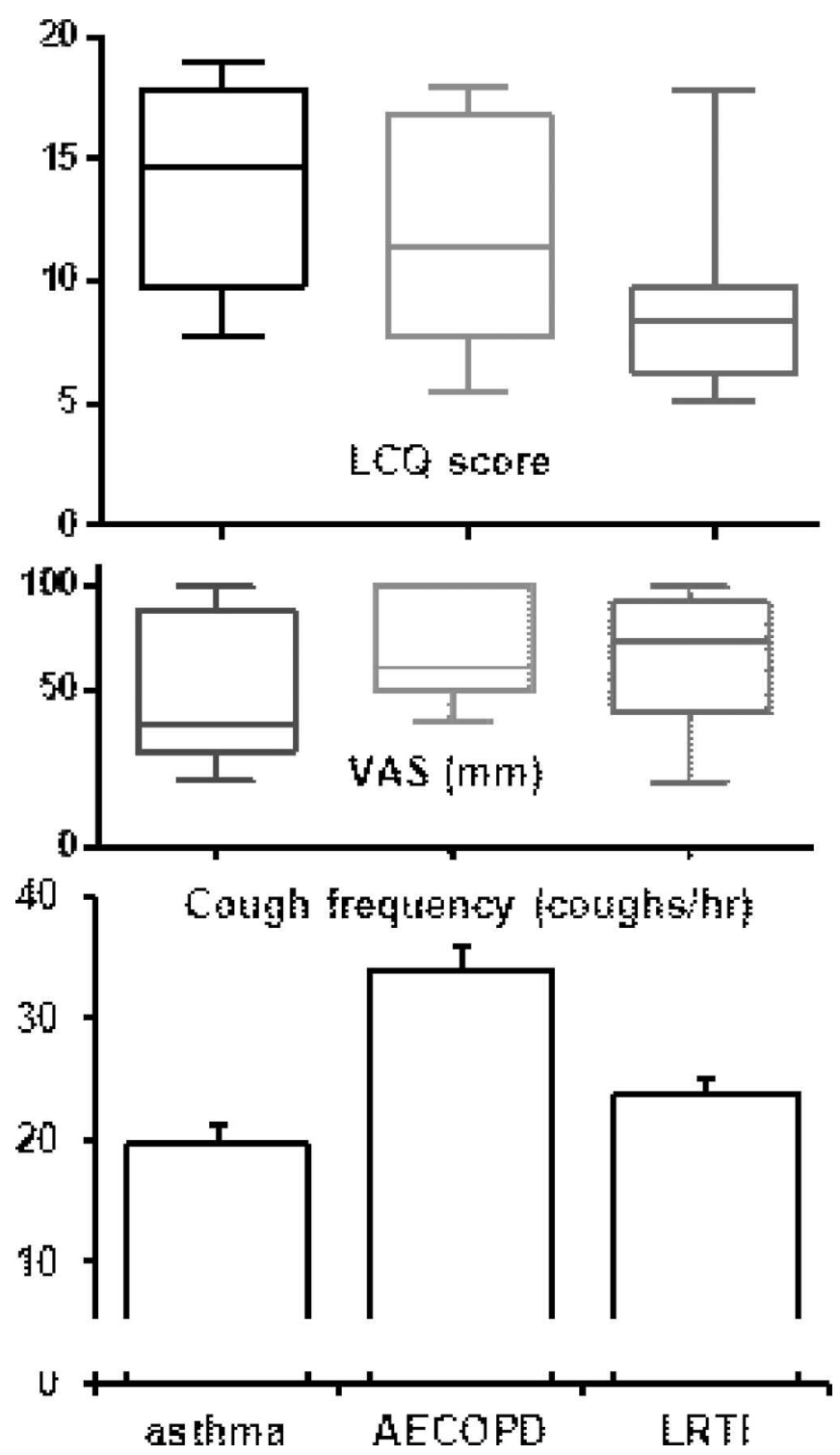

Abstract S29 Figure 1. Quality of life, subjective cough severity and 24-hour cough frequency in acute respiratory conditions Leicester Cough Questionnaire (LCQ) and cough visual analogue scale(VAS) data shown as median, range and interquartile range. Cough frequency shown as geometric mean and $\log _{10} \mathrm{SD}$. respiratory disease (LRTI). Quality of life was measured with the Leicester Cough Questionnaire (LCQ-acute), cough severity with a visual analogue scale (VAS) and 24-hour cough frequency with the Leicester Cough Monitor.

Results 40 patients were recruited within a median (interquartile range) of 1 (1-2.3) day ( ) of hospital admission. Median (IQR) age was 57 (41-71) and $63 \%$ were female. Geometric mean $\pm \log _{10} \mathrm{SD}$ cough frequencywas high: $19.7 \pm 1.36$, $33.8 \pm 2.02$ and $23.6 \pm 1.31$ coughs $/ \mathrm{h}$ for asthma $(\mathrm{n}=11)$, AECOPD ( $\mathrm{n}=15)$ and LRTI $(\mathrm{n}=14)$ respectively (Figure); median (IQR) cough bouts/24h: 81 (54-210), 148 (97-197) and 129 (67-197). There was no significant difference between disease groups in these values ( $p>0.05$ for all two-way comparisons). Diurnal variation and median numbers of coughs/ bout were similar between groups. The $48 \%$ of patients who were current smokers coughed more than non-smokers $(33.6 \pm 1.91$ vs $20.2 \pm 1.38$ coughs $/ \mathrm{h}, \mathrm{p}=0.07)$. No difference in cough frequency was detected amongst the $25 \%$ taking angiotensin converting enzyme inhibitors. Gender had no significant overall effect.

Median (IQR) VAS scores were 39 (32-86), 73 (53-100) and 82 (48-91) for asthma, AECOPD and LRTI respectively with no significant difference between them. Cough severity showed a significant correlation with 24-hour cough frequency overall (Spearman' coefficient $0.33, \mathrm{p}=0.05)$. LCQ-acute scores were lower for LRTI $(8.4 ; 6.4-9.5)$ than asthma $(14.7 ; 10.7-17.5) ; \mathrm{p}=0.01$ (Figure 1). Neither was significantly different from those for AECOPD (11.5; 8.5-15.6). Quality of life did not correlate with cough frequency (Spearman' coefficient $-0.13 ; \mathrm{p}=0.48$ ).

Conclusion Cough frequency in acute respiratory disease is high but with high variation. Cough frequency accounts for only part of morbidity in these conditions.

\section{S30 SALIVARY PEPSIN AS A BIOMARKER OF AIRWAY REFLUX IN IDIOPATHIC PULMONARY FIBROSIS - AN OBSERVATIONAL STUDY}

${ }^{1} \mathrm{JM}$ Dudziak, ${ }^{2} \mathrm{MG}$ Crooks, ${ }^{3} \mathrm{AD}$ Woodcock, ${ }^{3} \mathrm{PW}$ Dettmar, ${ }^{2} \mathrm{AH}$ Morice, ${ }^{2} \mathrm{SP}$ Hart; ${ }^{1}$ Academic Respiratory Medicine, Castle Hill Hospital, Cottingham, UK; ${ }^{2}$ Centre for Cardiovascular and Metabolic Research, Hull York Medical School, Hull, UK; ${ }^{3} R D$ Biomed Ltd, Castle Hill Hospital, Cottingham, UK

\subsection{6/thoraxjn-2013-204457.37}

Introduction and Objectives Current understanding of IPF proposes repetitive pulmonary epithelial injury with aberrant healing as a principal mechanism. Gastro-oesophageal reflux (GOR) and micro-aspiration of gastric contents may cause lung injury with subsequent fibrosis, and GOR is known to be prevalent in IPF patients. We assessed the feasibility of salivary pepsin measurement in IPF patients and investigated the temporal variability and relationship between salivary pepsin and symptoms.

Methods IPF patients collected saliva samples at multiple time points over the course of one day. Early morning, lunch- and dinner-time samples were analysed and compared with results from a historical control group of 100 healthy volunteers. Samples were analysed for the presence of pepsin using Peptest ${ }^{\mathrm{TM}}$ (RD Biomed Ltd). Patients were defined as pepsin positive if they had pepsin detectable in at least 1 saliva sample. The St George's Respiratory Questionnaire (SQRQ), Hull Airways Reflux Questionnaire (HARQ), and the REFLUX questionnaire were used to assess the relationship between pepsin positivity and symptoms.

Results All 21 IPF patients successfully provided saliva samples, of which 17 patients $(81 \%)$ were pepsin positive compared to 36 of 100 healthy volunteers $(36 \%), p=0.0004$. The proportion of 